\title{
Panta rhei in diagnosing rheumatic diseases
}

\section{Piotr Wiland}

Clinic of Rheumatology and Internal Medicine, Wroclaw University Hospital, Poland Department and Clinic of Rheumatology and Internal Medicine, Wroclaw Medical University, Poland

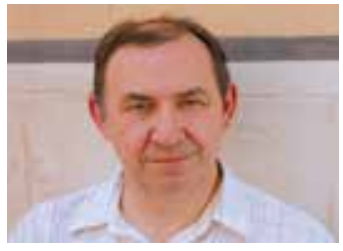

The quote ascribed to Heraclitus of Ephesus "Everything flows and nothing abides" (Greek "Panta rhei kai ouden menei") - is not particularly appreciated by physicians establishing the diagnosis in their patients. We typically want the diagnosis to be swift and accurate in individuals presenting with various constellations of signs and symptoms. This is hardly surprising, as science is dominated by the trend to stop the world in all its changeability and diversity. Rather than evaluating real-life phenomena, scientists tend to discuss abstracted models or still-frame scenes. But the world around us is not a static system. It constantly changes and moves, never standing still, and phenomena or things continuously interact with one another. The world of human diseases is no exception. Once established, a diagnosis does not always have to be a constant system.

This can happen under various circumstances. I do not refer here to cases of erroneous diagnosis caused by failure to carry out certain diagnostic tests, and incorrect analysis of the disease. There are circumstances when, because of mildly symptomatic course of the disease, the right diagnosis can only be established after a longer period of follow-up. A change in diagnosis can also occur following the introduction of new nosological entities such as pre-radiographic axial spondyloarthritis or IgG4-related disease.

Furthermore, an existing diagnosis can be modified after a change in disease classification criteria. This has happened particularly frequently with primary Sjögren's syndrome (pSS). Between 1986 and 2017, at least six sets of classification criteria for this disease were published. This fact should be taken into account when comparing groups of patients classified on the basis of different sets of criteria, and analyzing individual patients during a longer follow-up period. In a group of patients with suspected pSS, a total of 125 individuals had their diagnosis established on the basis of ACR/EULAR criteria
(2016) and AECG criteria (2002); furthermore 11 patients satisfied only the 2016 criteria, but there were no patients fulfilling solely the 2002 criteria. Consequently, an increase in the number of patients diagnosed with pSS concerned approximately $10 \%$ of patients [1]. The application of the 2016 criteria provided grounds for making the diagnosis in patients with pronounced systemic manifestations accompanied by absent or poorly manifested dry eyes and/or mouth.

The development of new classification criteria in recent years has been aimed primarily at improving detection at a relatively early stage of the disease, i.e. increasing the sensitivity of the criteria. This was also the rationale for the publication of new EULAR/ACR classification criteria for the diagnosis of rheumatoid arthritis (RA) in 2010. Among patients seen in routine clinical practice (without prior exclusion of other systemic and inflammatory diseases of joints), the sensitivity of the new criteria has indeed increased from $93 \%$ to $97 \%$ compared to the RA classification criteria from 1987, however their specificity has declined (55\% vs $76 \%$ ). This means that almost half (45\%) of the patients who, after careful observation, are ultimately diagnosed with a disease other than RA nevertheless meet the 2010 classification criteria [2].

Specificity and sensitivity may vary slightly depending on which population of patients is assessed and whether the rheumatologist has previously performed a selection in the group of patients presenting with swollen joints, and excluded other conditions. Nevertheless, the automatic application of the 2010 classification criteria in the population of patients with early arthritis may lead to overdiagnosis. During a longer follow-up period, such patients may be ultimately diagnosed with a different condition. For example, in another study conducted on a group of patients with very early arthritis, comparing the 1987 and 2010 criteria, 4 times more patients diagnosed with RA based on the 2010 criteria had a spontaneous remis-

Address for correspondence:

Piotr Wiland, Clinic of Rheumatology and Internal Diseases, Wroclaw University Hospital, 213 Borowska St., 50-556 Wrocław,

Poland, e-mail: pwiland1@gmail.com

Submitted: 24.06.2019; Accepted: 26.06.2019 
sion without the need to start DMARDs, compared to the patients fulfilling the 1987 criteria [3].

A change in diagnosis may also occur in patients with a rheumatic disease beginning at the developmental age and persisting into adulthood [4]. Based on a follow-up of more than 20 years, out of 131 patients with juvenile idiopathic arthritis (IIA) entering adulthood only $21 \%$ were unclassifiable in any adult rheumatic disease. This occurred particularly frequently in individuals with JA involving multiple joints at onset, and with RF-negative polyarthritis. With regard to RF-positive polyarthritis, $95.6 \%$ of patients could be classified as RA, while enthesitis-related arthritis (ERA) could be classified as spondyloarthritis (SpA). In cases of systemic-onset juvenile idiopathic arthritis (SOJIA) the patients were classified as having adult Still's disease (ASD) in adulthood, whereas in all patients with juvenile-onset psoriatic arthritis (PsA) the original diagnosis was sustained at an adult age.

Also, if a connective tissue disease (CTD) is diagnosed, there are situations where it may evolve into another CTD, especially in the presence of certain specific antibodies. In cases of primary Sjögren's syndrome, the presence of anti-citrulline antibodies may presage the evolution of the disease into rheumatoid arthritis (RA). During an 8-year follow-up in a group of patients with this disease and existing anti-citrulline antibodies, the diagnosis of RA based on the 1987 criteria could be established in $43.8 \%$ of the patients [5].

Mixed connective tissue disease (MCTD) is considered to be a separate nosological entity, with the obligatory diagnostic criterion of anti-U1RNP antibodies present in blood. Earlier studies suggested that the majority of patients with MCTD would evolve to a definite connective tissue disease (CTD), especially to systemic lupus erythematosus (SLE) or systemic sclerosis (SSc). More recent studies have shown that evolution is observed in less than one-third of patients. The results of 50 cases of MCTD from Mayo Clinic showed that evolution to other CTDs occurred with a 10-year rate of evolution of $8.5 \%$ for SLE, and $6.3 \%$ for SSc [7].

By definition, uncertainty in the diagnosis and evolution of the disease is particularly common in patients diagnosed with undifferentiated connective tissue disease (UCTD). The criteria for UCTD were proposed in 1999. Patients can be classified as UCTD if they meet the following criteria: a) signs and symptoms suggestive of connective tissue disease (CTD), without fulfilling the criteria for defined CTD, b) positive antinuclear antibodies on two separate measurements controls, and c) disease duration of at least 3 years. In recent years, following the development of new classification criteria for specific CTDs, the number of UCTD cases may decrease, and physicians providing care to such patients should be obliged to reconsider the diagnosis of CTD in the light of new classification criteria. After a mean follow-up of $11 \pm 3$ years in 98 patients with UCTD at baseline, $62 \%$ of the patients suffered from UCTD, $24 \%$ were in remission, and $14 \%$ developed definite CTD. High ANA titres or the presence of cytopaenias at baseline, as well as progression of nailfold-capillaroscopy pattern during follow-up, were the crucial factors associated with evolution to definite CTD [8].

In daily rheumatological practice, one should not approach a previously made diagnosis too routinely, especially when the patient's condition was diagnosed a considerable time ago, and clinical symptoms or laboratory test results have since changed their profile.

The author declares no conflict of interest.

\section{References}

1. Le Goff M, Cornec D, Jousse-Joulin S, et al. Comparison of 2002 AECG and 2016 ACR/EULAR classification criteria and added value of salivary gland ultrasonography in a patient cohort with suspected primary Sjögren's syndrome. Arthritis Res Ther 2017; 19: 269

2. Kennish L, Labitigan M, Budoff S, et al. Utility of the new rheumatoid arthritis 2010 ACR/EULAR classification criteria in routine clinical care. BMJ Open 2012; 2: e001117.

3. Cader MZ, Filer A, Hazlehurst J, et al. Performance of the 2010 ACR/EULAR criteria for rheumatoid arthritis: comparison with 1987 ACR criteria in a very early synovitis cohort. Ann Rheum Dis 2011; 70: 949-955.

4. Oliveira-Ramos F, Eusébio M, Martins FM, et al. Juvenile idiopathic arthritis in adulthood: fulfilment of classification criteria for adult rheumatic diseases, long-term outcomes and predictor of inactive disease, functional status and damage. RMD Open 2016; 2: e000304.

5. Payet J, Belkhir R, Gottenberg JE, et al. ACPA-positive primary Sjögren's syndrome: true primary or rheumatoid arthritis-associated Sjögren's syndrome? RMD Open 2015; 1: e000066.

6. Antunes M, Sciré CA, Talarico R, et al. Undifferentiated connective tissue disease: state of the art on clinical practice guidelines. RMD Open 2019; 4: e000786.

7. Ungprasert P, Crawson CS, Chowdhary VR, et al. Epidemiology of Mixed Connective Tissue Disease 1985-2014. A population based study. Arthritis Care Res 2016; 68: 1843-1848.

8. Garcia-González M, Rodriguez-Lozano B, Bustabad S, et al. Undifferentiated connective tissue disease: predictors of evolution into definite disease. Clin Exp Rheumatol 2017; 35: 739-745. 